\title{
Erratum: Thermal Transport in Crystals as a Kinetic Theory of Relaxons [Phys. Rev. X 6, 041013 (2016)]
}

\author{
Andrea Cepellotti® and Nicola Marzari
}

(C) (Received 18 September 2020; published 26 October 2020)

DOI: 10.1103/PhysRevX.10.049901

Equation (C7) in Appendix C contains a typographical error, in the form of an inverted inequality sign: the correct expression should be

$$
\frac{1}{k} \geq \frac{1}{k_{1}}+\frac{1}{k_{2}}
$$

The discussion in the paper on its relevance to Matthiessen rule is correct, and adheres to the inequality above.

We gratefully acknowledge Dr. Yan Li for bringing this to our attention. 